\title{
Localization in mobile wireless and sensor networks
}

Monica Nicoli ${ }^{1 *}$, Sinan Gezici ${ }^{2}$, Zafer Sahinoglu ${ }^{3}$ and Henk Wymeersch ${ }^{4}$

Accurate localization or tracking of wireless devices is a crucial requirement for many emerging location-aware systems. Fields of applications include search and rescue, medical care, intelligent transportation, locationbased billing, security, home automation, industrial monitoring and control, location-assisted gaming, and social networking. During the last few years, there have been intensive research activities in this area and various solutions have been investigated. The main trend now is toward the integration of heterogeneous technologies to ensure global coverage and high accuracy in all possible scenarios, leading to a seamless localization system available anywhere anytime.

While satellite-based navigation is well consolidated for open sky scenarios, localization in harsh environments (e.g., indoor or in urban canyons) is still an open issue that requires complementary wireless networks. Cellular systems, local/personal area networks, ad hoc, and wireless-sensor networks can be configured to support localization functionality. Indoor environments, however, are particularly challenging because of severe multipath and non-line-of-sight (NLOS) propagation. In this context, advanced signal processing algorithms must be employed in order to guarantee positioning robustness, such as NLOS identification and mitigation, fusion of data from different sources, and Bayesian methods to enclose any a priori information (e.g., dynamic models for mobile positioning). An important area of research is cooperative localization, which is expected to significantly improve both accuracy and coverage by exploiting all the available measurements on a peer-to-peer basis; efficient protocols and procedures have to be designed to minimize communication overheads and energy consumption. Measurement campaigns are essential for calibrating signal models and testing localization algorithms. A valuable tool for

\footnotetext{
* Correspondence: nicoli@elet.polimi.it

'Dipartimento di Elettronica e Informazione, Politecnico di Milano, Milan, Italy

Full list of author information is available at the end of the article
}

benchmarking algorithms is also provided by fundamental performance bounds, which are being actively analyzed as guidelines for the design of efficient positioning systems.

The objective of this special issue, which was promoted under the auspices of the EC Network of Excellence in Wireless Communications NEWCOM++ (in particular, the Work Package WPR.B on Localization and Positioning Techniques), was to gather recent advances in both signal processing and communications areas, for localization in mobile wireless and sensor networks. Articles were solicited on both experimental and theoretical aspects, including new positioning algorithms and methodologies, system design and configuration, performance analysis and measurement campaigns.

We received a total of 56 manuscripts addressing the above issues and challenges, of which 16 were selected for publication. Selection of each article was the result of a careful assessment by at least two (mostly three) independent reviewers with expertise on localization and wireless networking. Articles went through a minimum of two to a maximum of four revision phases before acceptance. Accepted articles belong to four main research areas: integration of positioning and communication functionalities, robustness to NLOS errors, indoor positioning, and localization in wireless sensor networks (WSNs).

The first group of articles deals with the interaction of positioning and communications at different layers of the protocol stack. Connectivity issues are studied in Gao et al., which considers the relation between distance and communication hops, accounting for the border effect and dependence problems, for a model that is more realistic than the traditional unit-disk graph model. A related problem is investigated in the study of Moragrega et al., which deals with location-aware cluster formation. The authors propose LACFA, a distributed network formation algorithm that significantly increases the probability of localization of sensors in a cluster-tree topology. On the physical layer, Schmeink et 
al. propose a channel parameter estimator for joint communications and positioning systems, using soft information concerning the parameter estimates. On the network layer, Kong et al. tackle the problem of localization and link detection for location-aware routing, with particular emphasis on NLOS links. The authors complement their theoretical work with an experimental evaluation with commercial transceivers, operating in the $2.4 \mathrm{GHz}$ ISM band.

The second group of articles focuses on the challenging task of localization with high position accuracy in NLOS environments. Huang et al. derive the CramérRao lower bound (CRLB) for WSN localization when position coordinates of the reference devices are subject to errors. They use a non-parametric kernel method to estimate the probability density function of the NLOS errors. The provided CRLBs can also be applicable to LOS cases after setting the NLOS error to zero. In another article, Mallat et al. propose two time-of-arrival estimators and show that the estimators achieve the baseband CRLB. A positioning algorithm that is robust to NLOS links is described in the article by Gholami et al. The authors first discuss the properties of projection onto convex sets (POCS) and outer approximation (OA) techniques for use in positioning, and then develop distributed positioning algorithms based on POCS and OA. Improvement of coverage and accuracy performance, especially in NLOS scenarios, can be provided by cooperation among nodes. In the article by Eren, graphical conditions that lead to unique localizability in cooperative networks with hybrid distance and bearing measurements are determined. The author also shows that the localization problem is solvable in linear time, and it is possible to reduce the required number of sensing links.

The next group of articles shifts the focus on localization in indoor environments. New techniques based on received signal strength (RSS) are presented, placing the emphasis on calibration of pathloss models and digital maps on experimental data. More specifically, Vanheel et al. present a test-bed for indoor localization in WSNs using RSS lateration. An automated method is proposed for calibration of the pathloss model and pre-processing of measured data in order to optimize the localization performance. The article by Oussar et al. deals with localization of GSM devices in domestic or office environments using a very large database of RSS fingerprints. Machine learning techniques are employed to extract the location information from online RSS measurements showing promising performance for room-level classification. The use of differential radio maps is proposed by Wang et al. to mitigate the effects of dynamic environments and accommodate different receiver gains. Particle filters (PFs) are employed to track moving targets using observation likelihoods obtained from the differential radio maps. Next two articles extend the analysis of Bayesian filtering with new results for indoor navigation. The first one, by Kaiser et al., presents a new motion model for pedestrians with a priori information on maps and floor plans. The model is derived from a diffusion algorithm that makes use of the principle of a source effusing gas and is exploited by a PF tracker for improving navigation performance. The second article, by Dhital et al., investigates experimentally the suitability of a number of Bayesian filtering techniques for indoor positioning by ultrawide band networks. The article also highlights the robustness of the cost-reference PF to model inaccuracies. Finally, the article by Callaghan et al. studies the feasibility of localization of a set of wireless nodes in a rich-scattering environment using signals received from ambient sources, without any knowledge of sources locations and transmitted waveforms. Pairwise distances are derived from cross correlations of the received signals using statistical methods assisted by multi-dimensional scaling. Tests on both simulated and real measurements in an office environment show localization accuracy of about $2 \mathrm{~m}$.

The last two articles, by Gustafsson et al. and Xaver et al., investigate localization algorithms for WSNs. In the first article, RSS-based localization is studied for realistic situations in which neither the emitted power nor the power law decay exponent are known. The authors first validate a model in the logarithmic scale, which is linear in the unknown nuisance parameters. Then, they develop a localization algorithm based on this model. The proposed algorithm can be useful in rapidly deployed networks consisting of a number of sensor nodes with low-bandwidth communications. In the second article, decentralized localization of an acoustic source is studied in a sensor network based on the underlying partial differential equation. An algorithm is proposed for the localization of multiple acoustic sources by employing decentralized particle filtering, which exploits the sparsity of the matrices in the statespace model. Also, a version of the maximum consensus algorithm is used to aggregate local posterior distributions from the clusters.

We would like to thank the authors of all submitted articles for considering our special issue for disseminating their study. We are also very grateful to the numerous reviewers who provided valuable and timely feedback to the authors. Their efforts were very helpful in improving the quality of the accepted articles. We would also like to thank the staff of Hindawi and SpringerOpen for their valuable assistance through the entire editing process, and the Editor-in-Chief of the journal, Prof. Luc Vandendorpe, for trusting us with this important assignment and helping us fulfil it successfully. Last but not least, we thank the members of 
NEWCOM++ for their collaboration in submitting highquality articles to this special issue.

Monica Nicoli

Sinan Gezici

Zafer Sahinoglu

Henk Wymeersch

\section{Author details}

'Dipartimento di Elettronica e Informazione, Politecnico di Milano, Milan, Italy ${ }^{2}$ Department of Electrical and Electronics Engineering, Bilkent University, Ankara, Turkey ${ }^{3}$ Mitsubishi Electric Research Labs, Cambridge, MA, USA

${ }^{4}$ Department of Signals and Systems, Chalmers University of Technology, Gothenburg, Sweden

Received: 23 August 2011 Accepted: 6 December 2011

Published: 6 December 2011

doi:10.1186/1687-1499-2011-197

Cite this article as: Nicoli et al: Localization in mobile wireless and sensor networks. EURASIP Journal on Wireless Communications and Networking 2011 2011:197.

\section{Submit your manuscript to a SpringerOpen ${ }^{\circ}$ journal and benefit from:}

- Convenient online submission

- Rigorous peer review

- Immediate publication on acceptance

- Open access: articles freely available online

- High visibility within the field

- Retaining the copyright to your article 